\title{
Risk Analysis of DP Incidents during Drilling Operations
}

\section{Zaloa Sanchez-Varelaa, David Boullosa-Falces ${ }^{b}$, Juan Luis Larrabe-Barrenab $^{b}$, Miguel Angel Gomez-Solaeche ${ }^{b}$}

This paper aims to present a method to determine the type of dynamic positioning (DP) incidents that have a more significant risk during drilling operations in the period 20072015 , according to the element or the type of failure that causes the DP system to fail. Two different classifications are made: 1) according to the element that produces the incident (which has been the traditional classification in the industry) and 2) according to the type of error that arises, the latter being an alternative classification proposed in this paper. The predictable financial losses for each level of severity are used to define the resulting consequences for each case. A risk analysis is performed with the data obtained, showing the potentially more dangerous incidents, either because of their higher number of occurrences or because their consequences are remarkable. According to the classification proposed, the main causes with the higher risk results were power and environmental, according to the traditional classification, and fault/failure. Thus, the power segment's combination of failures is the riskiest cause during the DP drilling operations.

\section{KEY WORDS}

$\sim$ Dynamic positioning

$\sim$ Offshore

$\sim$ Risk analysis

$\sim$ Drilling

a. University of Split, Faculty of Maritime Studies, Split, Croatia

e-mail: zsanchezv@pfst.hr

b. Faculty of Engineering in Bilbao, University of the Basque Country, Portugalete, Spain e-mail: david.boullosa@ehu.eus

doi: $10.7225 /$ toms.v10n01.006

This work is licensed under (cc) BY

Received on: Feb 24, 2021 / Revised on: Apr 9, 2021 / Accepted on: Apr 17, 2021 / Published: Apr 20, 2021

\section{INTRODUCTION}

A dynamic positioning (DP) system is an automation used in marine platforms and vessels. Data from wind, currents and ship's motions are taken from different sensors. After analysing them, a signal is sent to the thrusters and rudders to compensate for those movements. This procedure seeks two main goals: maintaining a given position or moving the vessel along a preset track.

This sophisticated system has been in use since the 1970s. Its many applications are primarily found in the offshore industry. The complexity and high accuracy requested for the different offshore operations make the dynamic positioning system a great asset for this sector.

However, rarely does such a sophisticated automated system always perform well. The study of the incidents reported by vessels is vital to discover any failures that could be corrected and to improve DP operation safety.

Various institutions, both governmental and professional, have dealt with these issues, and have contributed to the safety improvement of DP operations by publishing guidelines and circulars for the sector.

Among the groups that have more actively provided feedback information to the industry regarding safety in DP operations, two professional organisations should be emphasised: the International Marine Contractors Association (IMCA) (https://www.imca-int.com/) and the Marine Technology Society (MTS) (https://www.mtsociety.org/). Other organisations, such as the International Maritime Organisation (IMO) (https:// www.imo.org/), different classification societies or flag states base their guidelines on the IMCA and MTS documents.

The MTS is a professional organisation based in Washington DC, USA, whose aim is "to promote awareness, understanding, advancement and application of marine technology" (Marine 
probabilities for a set of variables (Ancione, Bragatto and Milazzo, 2020). In addition, the system theoretic process analysis (STPA) is used for analysing the dynamic behaviour of the systems, providing advantages over other traditional methods (Leveson et al. 2012).

Examples of the application of these methodologies to the field of dynamic positioning are given by Sulaman et al. (2019), Abrecht (2016), and Parhizkar et al. (2020), with different results.

\subsection{The use of Dynamic Positioning Systems in Drilling Operations}

Drilling operations take place over a wellhead. The DP system's primary purpose is to maintain the drilling vessel's position so that the riser/stack angle is close to zero, compensating for currents or tidal flow if necessary. This angle is the one measured between the riser (on the top) and the wellhead or lower marine riser package (LMRP) (Bray, 2018b).

To achieve this, a watch circle system is created for the Dynamic Positioning Operator (DPO) to monitor the vessel's movements. In normal conditions, the vessel will work within the green circle (as shown in Figure 1). Should there be an incident in which the vessel cannot maintain position, there will be an LoP (known as drift-off or drive-off) beyond the green circle. In this case, the blue advisory alarm will be raised, indicating a degrading status.

If the LoP continues beyond the yellow circle, the yellow alarm should be sounded, and emergency disconnection preparations should occur.

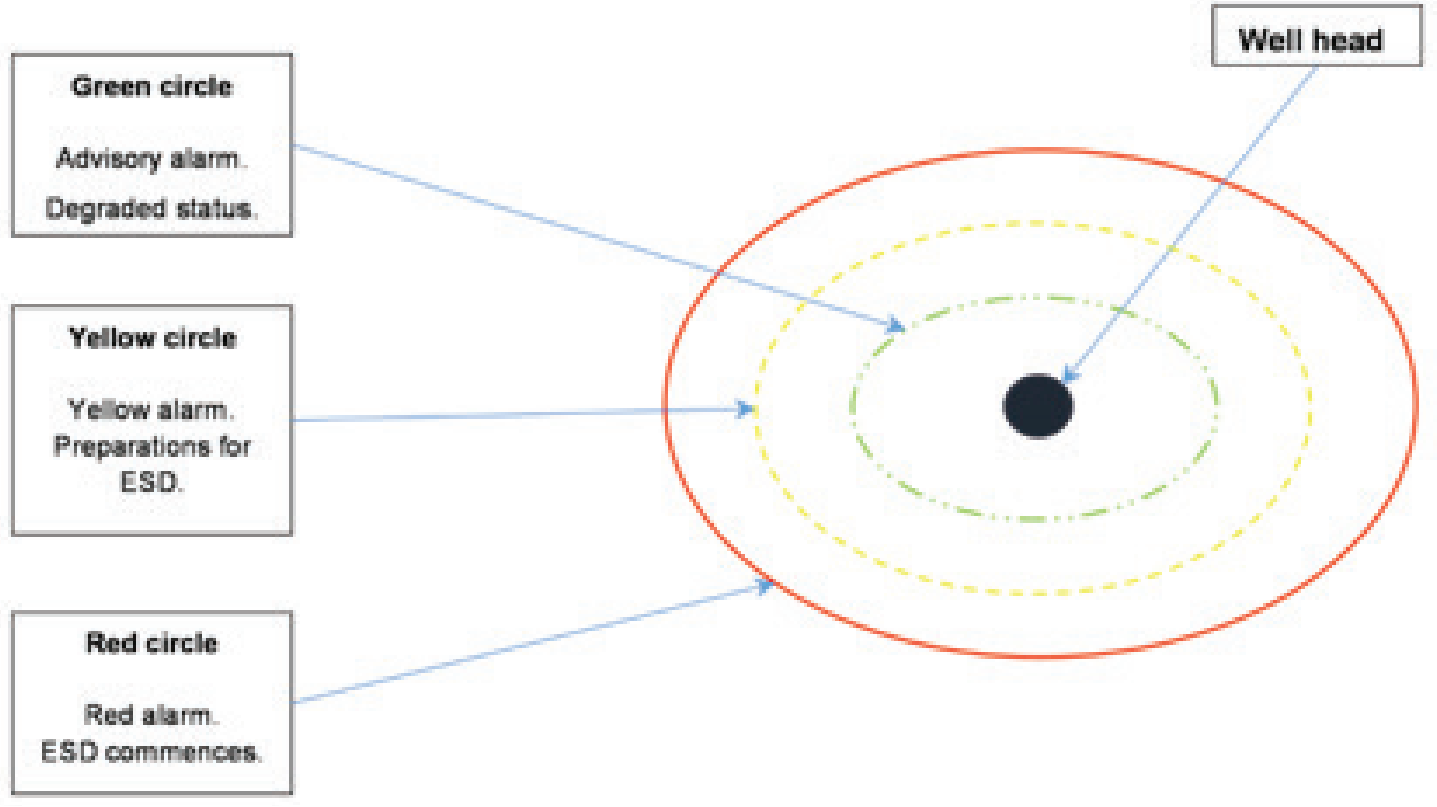

Figure 1.

DP watch circle (the LoP limits green, yellow, and red are not to scale).

Should the LoP continue beyond the red circle, the red alarm is raised, and an emergency controlled disconnection commences.

In a worst-case scenario, the LoP will not be stopped, and the vessel will surpass the red limit. In that case, emergency disconnection should start and the well be shut. However, if the vessel overpasses the physical limit, the riser would break, and the consequences would be catastrophic.

Some authors, like Chen, Moan and Verhoeven (2008) support the idea of determining the raddii of the circles based on the riser/stack angle; thus, the yellow circle will be set for an angle of $3^{\circ}$, and the red circle for a riser angle of $5^{\circ}$. This idea can be valid for shallow waters. As Bray (2018a) indicates, the tidal flow should be taken into account in deeper waters, compensating for its effect on the raiser.

However, the studies made in this field by authors like Weingarth (2006), Bhalla and Cao (2005), Quigley and Williams (2015), Adamson and Abrahamsen (2006), and Teixeira, Oshiro and Tannuri (2014) suggest that there are other factors to be taken into account (e.g. the presence of other objects in the surroundings), on a case-by-case basis. The MTS clearly states this in its DP Operations Guidance - Part 2 - Appendix 1 - MODUs (Marine Technology Society, 2012), where the Well Specific Operating Guidelines (WSOG) are implemented. The WSOG is 
a similar concept to the Activity Specific Operating Guidelines (ASOG) defined by IMCA in their Guidance on Operational Activity Planning (International Marine Contractors Association (IMCA), 2021). Here, the operational, environmental, and equipment performance limits for each location and activity are specified.

The main objective of this paper is to determine what kind of DP incidents are under a more prominent risk during drilling operations in the period 2007-2015, according to the element or the type of failure that causes the DP system to fail. Determining the kind of incident that is potentially more dangerous could help focus on the DP system segment with a more significant risk.

The secondary objectives are included in a statistical analysis of the incidents and add value to the main objective. Thus, checking the trends of the incidents during the study period is one of the secondary objectives.

This paper proposes an alternative causality categorisation based on the type of failure observed. This new categorisation is compared to the existing causality categorisation presented by the IMCA. The new categorisation is used to complement the traditional one based on the DP system segment in which the incident is triggered. Another secondary objective would be to determine with the help of a correlation table what DP segment is affected by what kind of mistake.
In addition, the secondary causes can add value to the description of the incidents. Knowing the distribution of the secondary causes and their relationship with the main causes will be another secondary objective of this research.

\section{METHODOLOGY}

\subsection{Database}

During the period 2007 to 2015, a total of 642 DP station keeping incidents were reported to the IMCA (International Marine Contractors Association (IMCA), 2009, International Marine Contractors Association (IMCA), 2010, International Marine Contractors Association (IMCA), 2011, International Marine Contractors Association (IMCA), 2012, International Marine Contractors Association (IMCA), 2015a, International Marine Contractors Association (IMCA), 2015b, International Marine Contractors Association (IMCA), 2015c, International Marine Contractors Association (IMCA), 2016a, International Marine Contractors Association (IMCA), 2016b) and distributed by years, as shown in Table 1.

Table 1.

Data about DP incidents reported each year (prepared by the authors, using data obtained from IMCA DP station keeping incidents, from 2012 to 2015.

\begin{tabular}{|c|c|c|c|c|c|c|}
\hline YEAR & $\begin{array}{l}\text { No. of vessels } \\
\text { reporting }\end{array}$ & $\begin{array}{l}\text { No. of vessels } \\
\text { reporting } 1 \\
\text { incident }\end{array}$ & $\begin{array}{l}\text { No. of vessels } \\
\text { reporting } 2 \\
\text { incidents }\end{array}$ & $\begin{array}{l}\text { No. of vessels } \\
\text { reporting } 3 \text { or } \\
\text { more incidents }\end{array}$ & $\begin{array}{l}\text { Total no. of } \\
\text { incidents }\end{array}$ & $\begin{array}{l}\text { Average } \\
\text { incidents per } \\
\text { vessel }\end{array}$ \\
\hline 2007 & 49 & 36 & 10 & 3 & 67 & 1.37 \\
\hline 2008 & 64 & 42 & 10 & 12 & 111 & 1.73 \\
\hline 2009 & 46 & 32 & 7 & 7 & 75 & 1.63 \\
\hline 2010 & 41 & 33 & 5 & 3 & 56 & 1.12 \\
\hline 2011 & 46 & 38 & 8 & 0 & 54 & 1.17 \\
\hline 2012 & 46 & 37 & 6 & 3 & 64 & 1.39 \\
\hline 2013 & 49 & 42 & 2 & 5 & 64 & 1.31 \\
\hline 2014 & 54 & 42 & 9 & 3 & 71 & 1.31 \\
\hline 2015 & 59 & 46 & 6 & 7 & 80 & 1.36 \\
\hline
\end{tabular}

The first step was to determine what operations were carried out when the incident happened. The event tree described the operations in progress, but this information was not uniform and had to be labelled and categorised by the team.

From the total DP incidents during the study period, the IMCA published 81 reported DP incidents that took place while drilling operations were in progress. However, a closer analysis led the research team to reject two of these incidents from the study. One of them referred to well intervention operations, so it was decided to remove it from the drilling DP incidents group of study. Although referring initially to a vessel engaged in drilling operations, the other incident had to do with an offshore 
supply vessel (OSV) undergoing cargo operations that had the DP incident. As the incident had not taken place on the vessel engaged in drilling operations, it was also decided to remove this incident from the study group.

All these incidents are presented in these publications with event-tree presentations. The data presented was then elaborated in a database, with the following entries: incident number, year, operation, main cause, secondary cause, comments, initiating event, and event description.

The incident reports published by the IMCA presented no clearly identified consequence. For each case, the event-tree stages were carefully read to retrieve the information for the consequence. The first classification was made by determining whether an LoP had happened or not. After that, the focus was put on the cases where an LoP was recorded. For these cases, it had to be determined whether the LoP had reached the red, yellow or red circle; then, the further LoP was taken for each case. When the consequence was not clear, the consensus was achieved by a meeting of the research team.

The consequences were then classified as an ordinal variable, according to the following classification:

1: $\quad$ no LoP;

2: $\quad$ LoP into the green circle;

3: LoP into the yellow circle;

4: $\quad$ LoP into the red circle.

The main and secondary causes were classified into one of the following categories: Computer, Electrical, Environmental, External, Human error, Power generation, References, Sensors and Thrusters/Propulsion. The IMCA uses this traditional classification of causes. It refers to the DP system component in which the incident was initiated without reflecting the error's nature. The research team decided to reflect this nature in an alternative causality categorisation. This alternative classification is related to the system's malfunction or error that leads to the DP system's degradation. In the following list, a description is given for each cause category:

Fault/failure occurs when a component stops working, their function cannot continue, and degradation of the system occurs. An alarm usually accompanies it.

Loss of signal occurs when a component cannot perform its function or it becomes limited due to a loss of the signal it needs to receive.

Procedures is the generic name given to the incidents caused by not following the given operational procedures. This includes, for example, pressing the wrong button in the DP station console.

Settings are assigned to the incidents in which the DP components are not set up correctly to perform to a desirable level of accuracy for the operation's needs.

Weather occurs when the wind force or the current speed, or both, are to blame for the incident.
First, a correlation between the traditional and the alternative classifications was performed using Pearson's residues (r) with the following formula:

$$
r_{i j}=\frac{e_{i j}-o_{i j}}{\sqrt{e_{i j}}}
$$

where $e_{i j}$ is the expected frequency value, and $o_{i j}$ is the observed frequency for different rows $i$ and columns $j$. The sign of the residue helps to determine whether the correlation is proportional or inversely proportional.

The contribution (c) of each pair to the Pearson's chi-square is then calculated as follows:

$c_{i j}=\frac{r_{i j}}{x^{2}}$

where $x^{2}$ represents the empirical chi-square obtained by the formula:

$x^{2}=\sum_{i, j} \frac{\left(e_{i j}-o_{i j}\right)^{2}}{e_{i j}}$

A more significant value of this contribution indicates a stronger correlation between the pair.

A descriptive statistic of the main causes was made for both causality classifications. It was considered noteworthy to present the different causalities per year and determine whether they were constant during the period.

To find out what main causes were more prone to have an LoP, a cross-table was made, taking into account the main causes and the variable that indicated whether an LoP had taken place or not. Since the number of observations was not very big $(n=79)$, a Montecarlo exact-test was used, creating 10,000 samples to determine the correlation for a p-value of 0.05 .

\subsection{Risk Classification}

Risk (R) is normally evaluated as a function of the severity of the possible consequences $(C)$ for a hazard and the probability of occurrence $(P)$ for that particular hazard:

$R=f(C, P)$ 
Both the consequences $(C)$ and the probability $(P)$ are functions of different parameters. However, it is common to simplify the function as the product of the consequences $(C)$ and the probabilities (P) (Kristiansen, 2005):

$R=C \cdot P$

As the incidents happening under the same cause can have a different consequence degree, an average consequence value is calculated.

In this study, $P$ is given by the number of cases registered for each consequence degree. This degree is determined by the watch circle surpassed during the incident.

The risk analysis was performed by multiplying for each different cause the number of incidents $(P)$ by the watch circle surpassed (C).

The operation's total risk can be obtained by adding up the risks obtained for each cause and consequence. The percentage of each cause contributing to the total risk is then considered and analysed.

The total risk was then calculated as the sum of the risks for each cause.

$R_{\text {total }}=\sum_{i=1}^{n} C_{i} \cdot P_{i}$

Being $\mathrm{n}$ the number of causes in the classification and $\mathrm{i}$ the number of different causes.

The value of the losses expected was estimated in the US dollars (USD) for each consequence. Considering the daily rates for different drilling units during the study period (information obtained from Seabreeze reports (Seabrokers Ltd, 2012-2015), the mean daily rate was calculated to be $279,010.00$ USD. This value was assigned to the incidents that reached the yellow circle. For the incidents without any LoP, the losses were zero as the operations continued without any downtime. The green and red circles' expected losses were estimated to be 55,000.00 USD and 1,000,000.00 USD respectively, as average quantities mentioned in conversations with experts in the field.

These expected losses were multiplied by the number of cases for each circle. Applying a bootstrapping technique with $\mathrm{R}$, we generated 5,000 samples with the same original distribution to obtain a bigger group of cases for the analysis. A boxplot diagram and a table with the central statistics were prepared from these samples, showing each category's risk distribution and calculating the differences in the central tendency measures using a Kruskall-Wallis test.

The distribution of the generated 5,000 samples was tested with a one-sample Kolmogorov-Smirnov non-parametric test to determine whether these distributions follow a normal or Poisson distribution.

\subsection{Statistical Software}

The statistical analysis of the database and the KolmogorovSmirnov test for the distribution of the risk were carried out using IBM SPSS Statistics software version 23.0 for Windows.

The risk analysis was performed using a bootstrapping technique with $\mathrm{R}$ studio.

\section{RESULTS}

\subsection{Annual Description of the Database}

Once the database was filtered for cases that took place while drilling operations were in progress, there were 79 cases, of which 45 cases ( $57 \%$ ) had a LoP and 32 (43\%) were able to maintain the position. When compared to the total incidents reported by the DP industry, which include other operations (such as diving, anchor handling, and cargo operations), there were 79 cases out of 642 , which is a significant percentage $(13 \%$, $\mathrm{p}$-value < 0.05)

There were 13 reports during the year 2007, only 3 cases in 2008, 6 cases in 2009, 7 cases in 2010, 8 cases in 2011, 6 cases in 2012,10 cases in 2013, 7 cases in 2014, and 19 cases in 2015. This data gives an average of 8.78 incidents per year.

The seriousness of the incidents per year was analysed, and the following results were obtained (as shown in Figure 2): in $2007,7.7 \%$ of the incidents had no LoP, while $46.2 \%$ reached the yellow circle, and $46.2 \%$ reached the red circle. In 2008, $33.3 \%$ of the incidents had no LoP, and all the incidents with LoP reached the red circle. In 2009, all the incidents had a LoP; $16.7 \%$ reached the yellow circle, and $83.3 \%$ reached the red circle. In 2010, 42.9 $\%$ of the incidents reached the green circle, and $57.1 \%$ reached the red circle. In 2011, $37.5 \%$ of the incidents had no LoP, $25 \%$ reached the yellow circle, and $37.5 \%$ reached the red circle. In $2012,66.7 \%$ of the incidents had no LoP, while $33.3 \%$ reached the red circle. In 2013, all the incidents remained in position. In 2014, $42.9 \%$ of the incidents had no excursion, while $28.6 \%$ reached the yellow circle, and $28.6 \%$ reached the red circle. In $2015,63.2 \%$ of the incidents had no LoP, $5.3 \%$ reached the green circle, and $31.6 \%$ reached the yellow circle. 


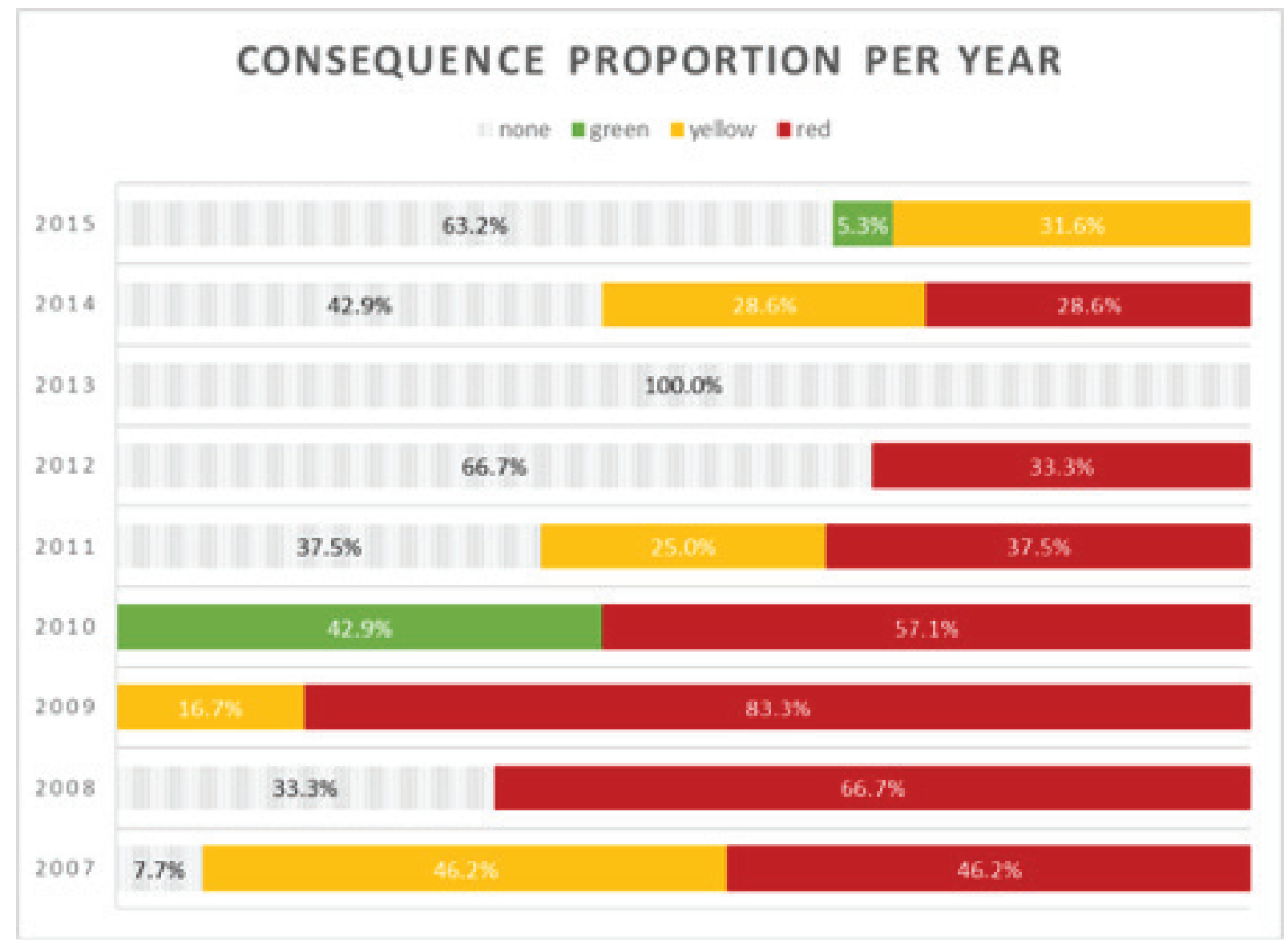

Figure 2.

Consequence proportion per year.

\subsection{The Relation Between Both Causality Classifications}

The traditional classification takes into account the element of the DP system that fails, while the alternative takes into account the nature of the failure. Thus, crossing both tables, the following information was obtained:

The main computer causes correspond in $50 \%$ of the cases to faults/failures, in $20 \%$ of the cases to loss of signal and procedures respectively, and $10 \%$ to settings. The main electrical cause is related to the settings (67\% of the cases) and fault/failure (33\%). Environmental causes are always related to weather, the same as main external causes, $100 \%$ in both cases. Human causes are related to procedures ( $83 \%$ of the cases), $8 \%$ relate to fault/failure, and an extra $8 \%$ to settings. Power has a great relationship with fault/failure (94\%), while the rest relates to settings. References and Sensors cause incidents when they lose their signals (100\% each). Thrusters are related to fault/ failure in $79 \%$ of the cases, while the rest $(21 \%)$ has to do with the signal loss. This cross-table is shown in Table 2.

When performing a chi-square with Monte Carlo exact test, we obtain that both systems are correlated ( $p$-value 0 ).

From the table of percentages of contribution to the chisquare, it can be observed that the most apparent correlations exist between Weather and Environment (23.2\%), Human and Procedures (19.1\%), and References and Loss of signal (12.6\%). The residues indicate that these relations are positive correlations.

The same methodology is applied to the secondary causes, and its results are shown in Table 3 . The more prominent correlations appear to be again between weather and environmental (27.2\%) and human cause and procedures (14.8 $\%)$. 
Table 2.

Cross-table showing the Pearson residues and contributions to the chi-square for the correlations between traditional and alternative main causes $(n=79)$.

\begin{tabular}{llllll}
\multirow{2}{*}{$\begin{array}{l}\text { Main cause } \\
\text { traditional }\end{array}$} & \multicolumn{5}{c}{ Main cause alternative } \\
\cline { 2 - 6 } & Fault/Failure & Loss of signal & Procedures & Settings & Weather \\
\hline Computer & $0.3(0.1 \%)$ & $0.7(0.2 \%)$ & $0.4(0.1 \%)$ & $0.5(0.1 \%)$ & $-1.5(1.2 \%)$ \\
\hline Electrical & $-0.3(0.0 \%)$ & $-0.6(0.2 \%)$ & $-0.7(0.2 \%)$ & $4.2(9.0 \%)$ & $-0.8(0.4 \%)$ \\
\hline Environmental & $-2.70(3.8 \%)$ & $-1.47(1.1 \%)$ & $-1.61(1.3 \%)$ & $-1.04(0.6 \%)$ & $6.67(23.2 \%)$ \\
\hline External & $-0.66(0.2 \%)$ & $-0.36(0.1 \%)$ & $-0.39(0.1 \%)$ & $-0.25(0.0 \%)$ & $1.62(1.4 \%)$ \\
\hline Human Error & $-1.83(1.7 \%)$ & $-1.23(0.8 \%)$ & $6.06(19.1 \%)$ & $0.28(0.0 \%)$ & $-1.65(1.4 \%)$ \\
\hline Power generation & $3.21(5.4 \%)$ & $-1.47(1.1 \%)$ & $-1.61(1.3 \%)$ & $-0.07(0.0 \%)$ & $-1.97(2.0 \%)$ \\
\hline References & $-1.31(0.9 \%)$ & $4.91(12.6 \%)$ & $-0.78(0.3 \%)$ & $-0.50(0.1 \%)$ & $-0.95(0.5 \%)$ \\
\hline Sensors & $-0.66(0.2 \%)$ & $2.45(3.1 \%)$ & $-0.39(0.1 \%)$ & $-0.25(0.0 \%)$ & $-0.48(0.1 \%)$ \\
\hline Thruster/Propulsion & $2.03(2.1 \%)$ & $0.92(0.4 \%)$ & $-1.46(1.1 \%)$ & $-0.94(0.5 \%)$ & $-1.79(1.7 \%)$ \\
\hline
\end{tabular}

Table 3.

Cross-table showing the Pearson residues and contributions to the chi-square for the correlations between traditional and alternative main causes $(n=34)$.

Secondary cause traditional

Secondary cause alternative

\begin{tabular}{llllll} 
traditional & Fault/Failure & Loss of signal & Procedures & Settings & Weather \\
\hline Computer & $(0.2 \%)$ & $(0.1 \%)$ & $(0.4 \%)$ & $-0.65(1.6 \%)$ & $(0.2 \%)$ \\
\hline Electrical & $-0.65(1.6 \%)$ & $(0.2 \%)$ & $(0.8 \%)$ & $-0.29(0.2 \%)$ & $(0.3 \%)$ \\
\hline Environment & $(0.7 \%)$ & $(0.2 \%)$ & $(1.2 \%)$ & $(1.4 \%)$ & $-1.53(27.2 \%)$ \\
\hline External & $(1.0 \%)$ & $(0.3 \%)$ & $(1.6 \%)$ & $-1.29(6.5 \%)$ & $(0.6 \%)$ \\
\hline Human Error & $(2.2 \%)$ & $(0.7 \%)$ & $-1.89(14.8 \%)$ & $2.18(2.0 \%)$ & $(1.4 \%)$ \\
\hline Power generation & $-1.22(7.0 \%)$ & $(0.4 \%)$ & $0.47(0.2 \%)$ & $0.76(0.5 \%)$ & $(0.8 \%)$ \\
\hline References & $(0.5 \%)$ & $(0.2 \%)$ & $-0.41(0.4 \%)$ & $(1.0 \%)$ & $-0.76(3.4 \%)$ \\
\hline Sensors & $(1.0 \%)$ & $-0.76(3.4 \%)$ & $(1.6 \%)$ & $-0.92(2.4 \%)$ & $(0.6 \%)$ \\
\hline Thruster/Propulsion & $-0.92(3.2 \%)$ & $-0.76(3.4 \%)$ & $(1.6 \%)$ & $0.41(0.2 \%)$ & $(0.6 \%)$
\end{tabular}

\subsection{The Relation Between Main and Secondary Causes}

The relationship between the main and secondary causes was studied with a cross table, finding out that the main cause Reference has in $75 \%$ of the cases a secondary cause. In contrast, External and Sensor main causes have no secondary causes at all. The analysis of the $p$-values showed that there were no significant distributions.

The main cause Computer, when having a secondary cause, is related to Human error in $80 \%$ of the cases, and Environmental cause in $20 \%$. The Electrical cause, when having a secondary cause is always related to Human error. The main Environmental causes have secondary causes: Sensors, References, Power (29\% each) or Human (14\%). The main causes related to Human error may have a wide variety of secondary causes: mainly External (33 $\%)$, but also Sensors, Power, Human and Computer (17\% each) contribute to them. Power's main causes can have a secondary cause related to Thrusters in $38 \%$ of the cases, Power (25\%), Electrical, External, and Sensors (13\% each). When having a secondary cause, with Reference systems it can be Human (67\%) or Environmental (33\%). Thruster main causes have as secondary causes, equally distributed, Thrusters, External, Environmental or Electrical. 
When the same comparison is made using the alternative classification, we obtain that Weather, Fault/Failure and Loss of signal are the main causes with more probability of having a secondary cause $(61 \%, 62 \%$ and $60 \%$, respectively). Settings have a probability of $40 \%$, and Procedures have a secondary cause in $33 \%$ of the cases. The p-values indicate that there is not enough evidence to accept the above trends as significant.

When there is a secondary cause, the distribution can be described as follows: when the main cause is Fault/Failure, then the secondary cause can be another Fault/Failure (31 $\%)$, Procedures or Settings ( $23 \%$ each), Loss of signal (15\%) or Weather (8\%). The secondary cause can be Procedures (50\%), and Settings or Weather ( $25 \%$ each) for the Loss of signal. When the main cause is Procedures, Settings account for $63 \%$ of the cases as secondary cause, while again Procedures happen in $38 \%$ of the cases. For main cause Settings, equally distributed, there are Fault/Failure, Settings and Weather as secondary causes (33\% each). When the main cause is Weather, the secondary cause could be Settings in $43 \%$ of the cases, Procedures (29\%), Weather or Fault/Failure (14\% each). The p-values indicate there is not enough evidence to extrapolate conclusions within a $95 \%$ confidence interval.

\subsection{Frequency}

\subsubsection{Traditional Classification}

The main causes with higher frequency were Environmental and Power, each of them happened 17 times during the study period; both causes together equal $43 \%$ of the total causalities. Incidents caused by problems with Thrusters happened in 14 cases, which means $17.7 \%$ of the total causes. Human errors caused 12 incidents during the period (15.2\% of the total), and Computers were to blame on ten occasions (12.7\%). The rest of the incidents had a lower frequency: References in four cases (5.1 $\%)$, Electrical in three cases (3.8\%), External and Sensors in one case each, (1.3\% each).

There were 34 incidents for which a secondary cause was defined. In 9 cases, the secondary causes were defined as Human errors $(26.5 \%)$, in 5 cases they were due to Power (14.7\%), 4 cases each $(11.8 \%)$ due to External factors, Sensors, and Thrusters. Due to secondary Environmental causes, we had 3 cases (8.8\%). For Reference and Electrical causes there were 2 cases (5.9\%). There was only 1 computer secondary cause (2.9\%).

\subsubsection{Alternative Classification}

For the main causes in the alternative classification, Faults and Failures happened more frequently, in 34 incidents, i.e. almost half of the incidents ( $43 \%$ of the total number of incidents). Weather was to blame in 18 incidents (22.8\%), and non-followed
Procedures lead to 12 incidents (15.2\%). Loss of signal happened in 10 incidents $(12.7 \%)$, and Settings were wrong and lead to an incident on 5 occasions (6.3\%).

The secondary cause with the highest frequency is Settings problems (12 cases or $35.3 \%$ ), followed by procedures (10 cases or $29.4 \%)$. Fault/failure appear in 6 cases (17.6\%), Weather in 4 cases (11.8\%) and the lowest frequency corresponds to Loss of signal, with 2 cases (5.9\%).

\subsection{Consequences}

\subsubsection{Traditional Classification}

When crossing the main causes with the LoP, it was found out that for the Power causes there was a LoP in 12 out of 17 incidents (70.6\%). This high frequency for a LoP was also found in Computer incidents (60\%), Environmental incidents (64.7\%) and Human errors (66.7\%). Thruster incidents presented a higher frequency for non-LoP incidents, as 11 out of the 14 incidents $(78.6 \%)$ resulted in no deviation from the wellhead. Cases with Sensors as main cause had no LoPs, so they are not shown in the following results.

Human error cases had a more significant percentage of LoP beyond the red circle (5 cases, $62.5 \%$ ), while only 1 case overpassed the green circle (12.5\%), and 2 cases passed the yellow circle (25\%). Environmental causes had 1 (9\%) case passing the green circle, 5 cases ( $45.5 \%$ ) passing the yellow circle and 5 cases ( $45.5 \%$ ) passing the red circle.

A chi-square test performed with a Monte Carlo exact test shows that the correlations between the causes and the consequences are not significant.

Regarding the secondary causes, those incidents in which there was an external secondary cause have a more significant probability of having a LoP (100\%), followed by Power (80 \%), Thrusters (75\%) and Human errors (67\%). Sensor secondary cause has a more significant probability of not having a LoP (75 $\%)$. The analysis of the $p$-values showed that the value for the external secondary cause could be taken as significant ( $p$-value $<0.05)$.

Out of the 25 LoP cases, the distribution of the watch circle reached is as follows: Power and External causes have the most significant probability to surpass the red watch circle (75\%), while human causes will have $50 \%$ of possibilities of surpassing the red circle and $33 \%$ of surpassing the yellow circle.

The chi-square test with Monte Carlo exact test does not show enough evidence for accepting these results. However, the Goodman and Kruskall tau shows the dependency of the secondary cause as significant.

When analysing the more significant contribution to the chi-square, we obtain the following results, as shown in Table 4. 
Table 4.

Cross-table showing the Pearson residues and the contributions to the chi-square (percentage in brackets) for the correlations between traditional secondary causes and watch circle reached.

Secondary cause (traditional)

Watch circle

\begin{tabular}{lllll}
\hline Computer & $-0.51(1 \%)$ & $-0.24(0 \%)$ & $-0.51(1 \%)$ & $0.92(4 \%)$ \\
\hline Electrical & $0.65(2 \%)$ & $-0.34(1 \%)$ & $0.65(2 \%)$ & $-0.91(4 \%)$ \\
\hline Environmental & $-0.89(4 \%)$ & $1.96(20 \%)$ & $0.23(0 \%)$ & $-0.21(0 \%)$ \\
\hline External & $-1.03(5 \%)$ & $-0.49(1 \%)$ & $-0.06(0 \%)$ & $1.05(6 \%)$ \\
\hline Human Error & $0.40(1 \%)$ & $0.65(2 \%)$ & $-0.25(0 \%)$ & $-0.37(1 \%)$ \\
\hline Power generation & $-0.28(0 \%)$ & $-0.54(2 \%)$ & $-0.28(0 \%)$ & $0.66(2 \%)$ \\
\hline References & $-0.73(3 \%)$ & $-0.34(1 \%)$ & $0.65(2 \%)$ & $0.19(0 \%)$ \\
\hline Sensors & $1.89(18 \%)$ & $-0.49(1 \%)$ & $-1.03(5 \%)$ & $-0.50(1 \%)$ \\
\hline Thruster/Propulsion & $-0.06(0 \%)$ & $-0.49(1 \%)$ & $0.91(4 \%)$ & $-0.50(1 \%)$
\end{tabular}

It is easy to see that those incidents that have Environment as a secondary cause are prone to stay within the green circle, while the incidents happening with Sensors as a secondary cause usually do not have any LoP.

\subsubsection{Alternative Classification}

The analysis of the causes that more frequently ended in LoP gave the following results: Procedures were the causes of an evident higher frequency of LoP with 9 cases out of 12 (75\%). The weather also had a higher frequency for LoPs, with 12 out of 18 cases $(66.7 \%)$. The Fault/Failure has 18 cases in which there was no LoP, and in 16 cases there was an LoP (47.1\%). For Loss of signal, very similar results were obtained, with 5 cases out of 10 having no LoP and 4 cases ( $40 \%$ ) having it. The p-values calculated showed that these percentages were not significant.
Among the 45 cases where an LoP took place, the distribution of watch circles that the unit had surpassed as a maximum was studied. For the cases due to a Fault or Failure, many of them surpassed the red circle (10 cases, $63 \%)$. In Weather, 1 case surpassed the green circle, 5 (42\%) the yellow circle and 6 (50\%) the red circle. Procedures surpassed the red circle in $56 \%$ of the cases.

A chi-square test with Monte Carlo exact test indicates that there is not enough evidence to extrapolate these results with a $95 \%$ confidence.

The highest probability of LoP exists for secondary loss of signal and weather causes (100\%), followed by settings (75\%) and procedures (70\%). For Fault and failure, the probability of a LoP is $50 \%$.

Within the 25 cases with a LoP, the reached watch circle is classified into different secondary categories. Settings have

Table 5 .

Cross-table showing the Pearson residues and the chi-square contributions for the correlations between alternative secondary causes and watch circle reached.

Secondary cause (alternative)

Fault/Failure

Loss of signal

Procedures

Settings

Weather

\begin{tabular}{llll}
\hline \multicolumn{4}{l}{ Watch circle } \\
\hline none & green & yellow & red \\
\hline$-0.72(15 \%)$ & $-0.59(4 \%)$ & $-0.47(3 \%)$ & $-0.30(1 \%)$ \\
\hline $0.22(1 \%)$ & $-0.34(1 \%)$ & $0.65(5 \%)$ & $0.19(0 \%)$ \\
$-0.10(0 \%)$ & $0.54(3 \%)$ & $0.22(1 \%)$ & $-0.55(4 \%)$ \\
$-1.03(13 \%)$ & $-0.84(9 \%)$ & $-0.10(0 \%)$ & $0.48(3 \%)$ \\
\hline
\end{tabular}


6 cases (67\%) that reach the red circle and 3 (33\%) the yellow circle. The percentages are the same for Fault/Failure, with 2 cases passing the red circle and one the yellow circle. Procedures have 3 cases ( $43 \%$ ) reaching the red circle, 3 cases ( $43 \%$ ) the yellow and 1 case (14\%) the green circle. Finally, incidents with secondary cause Loss of signal reached the red circle (50\%) and the other case reached the yellow circle (50\%). The chi-square with Monte Carlo exact test shows that the results could be extrapolated with a $95 \%$ confidence.

When the data is ordered and the chi-square contributions are determined, we obtain that the secondary cause weather incidents usually stay within the green circle. At the same time,
Fault/Failure is more prone to not having any LoP. These results are shown in Table 5.

\subsection{Risk Analysis}

Estimating the expected losses to be 55,000.00 USD for the incidents in which the green circle was reached, 279,010.00 USD for the incidents reaching the yellow circle and 1,000,000.00 USD for the incidents surpassing the red circle, the qualitative risk analysis of the main causes was obtained for the different causes.

The overall risk per year is shown in Figure 3.

Figure 3.

The sum of the overall risks per year is presented in this graphic. The highest risk corresponds to 2007. Since then, the trend line is clearly showing the risk is reducing as the years pass.

\subsubsection{Traditional Classification}

The total risk is calculated to be 28.9 million USD for the period of study of 9 years, which means that the expected losses are 3.2 million USD per year.

The contribution of each different cause to the absolute risk is then calculated as a percentage: Computer $13 \%$, Electrical 4
\%, Environmental $22 \%$, External $4 \%$, Human error $19 \%$, Power generation $29 \%$, References $1 \%$, Sensors $0 \%$, and Thruster/ Propulsion $8 \%$. Figure 4 represents the expected losses with a boxplot and a median graph. In Table 6, the main statistic parameters for the distribution are shown. 
Table 6 .

Statistic parameters for expected losses of each traditional cause category. (Prepared by authors using R Studio, with 5,000 samples generated using the bootstrapping technique).

\begin{tabular}{|c|c|c|c|}
\hline & & $\begin{array}{l}\text { Mean } \\
\text { (million USD) }\end{array}$ & $\begin{array}{l}95 \% \text { confidence interval } \\
\text { (million USD) }\end{array}$ \\
\hline \multirow{9}{*}{$\frac{. \frac{5}{\pi}}{\sum}$} & Computer & $3.85 \pm 1.77$ & $3.81-3.90$ \\
\hline & Electrical & $1.04 \pm 1.00$ & $1.02-1.07$ \\
\hline & Environmental & $6.48 \pm 2.20$ & $6.42-6.54$ \\
\hline & External & $1.02 \pm 1.01$ & $0.99-1.05$ \\
\hline & Human Error & $5.56 \pm 2.19$ & $5.50-5.62$ \\
\hline & Power generation & $8.37 \pm 2.51$ & $8.30-8.44$ \\
\hline & References & $0.34 \pm 0.29$ & $0.33-0.35$ \\
\hline & Sensors & 0.00 & $0.00-0.00$ \\
\hline & Thruster/Propulsion & $2.28 \pm 1.42$ & $2.24-2.32$ \\
\hline \multirow{9}{*}{ 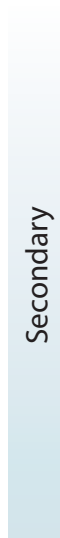 } & Computer & $1.02 \pm 0.99$ & $0.99-1.05$ \\
\hline & Electrical & $0.28 \pm 0.27$ & $0.27-0.29$ \\
\hline & Environmental & $1.33 \pm 1.03$ & $1.30-1.35$ \\
\hline & External & $3.27 \pm 1.67$ & $3.22-3.32$ \\
\hline & Human Error & $3.60 \pm 1.67$ & $3.56-3.65$ \\
\hline & Power generation & $3.27 \pm 1.68$ & $3.22-3.31$ \\
\hline & References & $1.28 \pm 1.00$ & $1.25-1.31$ \\
\hline & Sensors & $1.04 \pm 0.98$ & $1.01-1.06$ \\
\hline & Thruster/Propulsion & $1.59 \pm 1.06$ & $1.56-1.62$ \\
\hline
\end{tabular}

Regarding the secondary causes of the traditional classification, and following the same methodology, the following values were obtained: Computer $6 \%$, Electrical $2 \%$, Environmental $8 \%$, External $20 \%$, Human error $22 \%$, Power $20 \%$, References $8 \%$, Sensors $6 \%$, Thruster/Propulsion $10 \%$. All these results are graphically represented in Figure 4.

Similarly, the table and boxplot were generated for the secondary causes, showing the risk is 3.60 million USD for Human errors and 3.27 million USD for External and Power causes each.

A Kruskall Wallis non-parametric test was used for the distribution comparison, showing statistically significant differences among the main causes. For the secondary causes, the same test showed no significant differences between Power and External causes ( $p$-value $>0.05$ ), but the rest of the causes have a significantly different distribution.

The different causes do not follow a predetermined distribution after performing the one-sample KolmogorovSmirnov non-parametric test; they are neither normal nor Poisson; all the data has a positive skewness and is generally leptokurtic. 


\section{RISK PERCENTAGES OVER TOTAL}

MAIN TRADITIONAL CAUSES
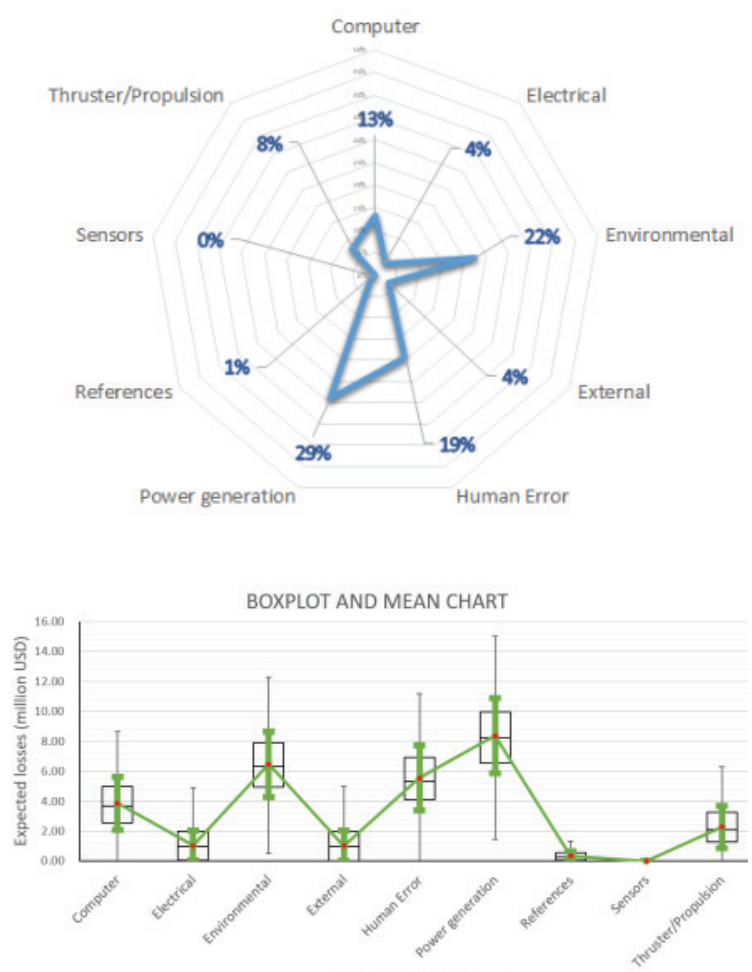

Main Traditional causes
RISK PERCENTAGES OVER TOTAL SECONDARY TRADITIONAL CAUSES
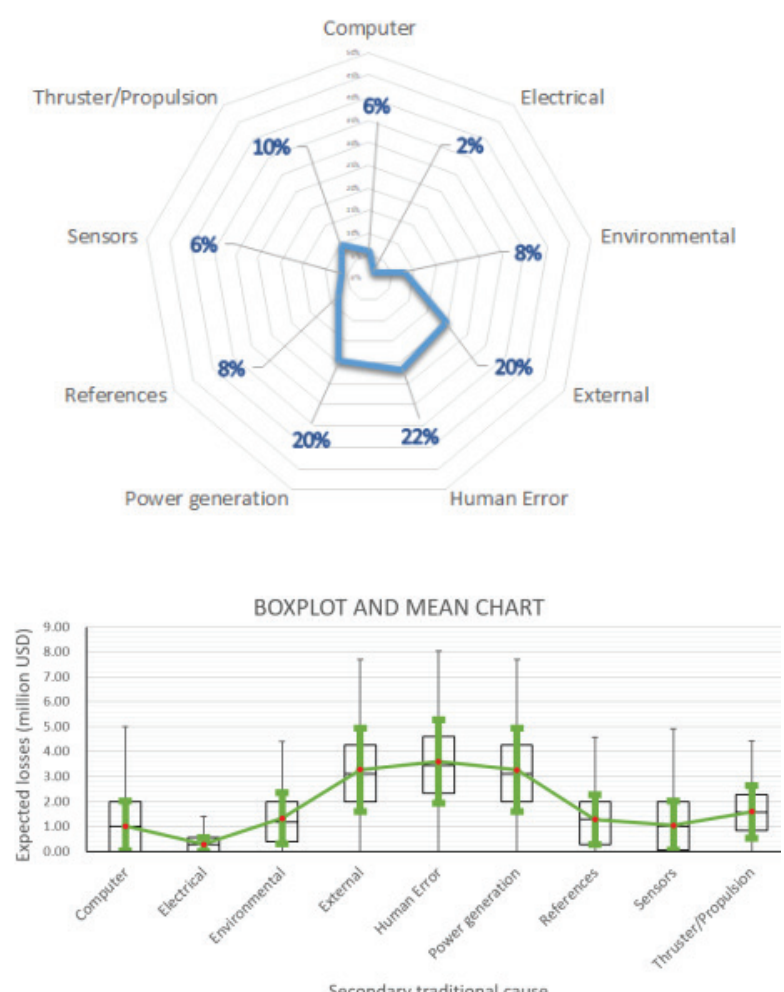

Figure 4

Top: Risk percentages for main traditional causes (left) and traditional secondary causes (right). Bottom: Boxplot and mean chart (showing standard deviations) for expected losses of main traditional causes (left) and traditional secondary causes (right).

\subsubsection{Alternative Classification}

By applying the same methodology as above for the alternative classification, the results obtained show that the distribution of the total risk (28.9 million USD) is as follows: Fault/ Failure $40 \%$, Loss of signal $5 \%$, Procedures $20 \%$, Settings $8 \%$ and Weather $26 \%$. The statistic parameters are shown in Table 7.

The higher expected losses for main causes are Fault/ Failure with an average risk of 11.64 million USD, while Weather and Procedures have 7.48 and 5.91 million USD risk for the period.

The secondary causes obtained the following values for quantitative risk: Fault/Failure $14 \%$, Loss of signal $8 \%$, Procedures $23 \%$, Settings $41 \%$ and Weather $14 \%$. The results for both main and secondary causes are represented in Figure 5 .
Regarding the secondary causes, Settings with 6.90 million USD is the cause with the higher risk, followed by Procedures with 3.92 million USD.

The Kruskall-Wallis test shows that all the distributions are significantly different among the different causes, both for main and secondary causes.

In the same way as for the traditional causes, the Kolmogorov-Smirnov non-parametric test results show that the distribution of the risk for the different causes is neither normal nor Poisson; here also all the data has a positive skewness and is generally leptokurtic. 
Table 7.

Statistic parameters for expected losses of each alternative cause category. (Prepared by authors using R Studio, with 5000 samples generated using the bootstrapping technique).

\begin{tabular}{|c|c|c|c|}
\hline & & $\begin{array}{l}\text { Mean } \\
\text { (million USD) }\end{array}$ & $\begin{array}{l}95 \% \text { confidence interval } \\
\text { (million USD) }\end{array}$ \\
\hline \multirow{5}{*}{$\frac{.5}{\sum^{\pi}}$} & Fault/Failure & $11.64 \pm 2.94$ & 11.56- 11.72 \\
\hline & Loss of signal & $1.59 \pm 1.06$ & $1.56-1.62$ \\
\hline & Procedures & $5.91 \pm 2.21$ & $5.84-5.97$ \\
\hline & Settings & $2.34 \pm 1.43$ & $2.30-2.38$ \\
\hline & Weather & $7.48 \pm 2.40$ & $7.42-7.55$ \\
\hline \multirow{5}{*}{ 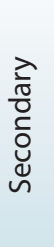 } & Fault/Failure & $2.28 \pm 1.39$ & $2.24-2.32$ \\
\hline & Loss of signal & $1.26 \pm 1.01$ & $1.23-1.29$ \\
\hline & Procedures & $3.92 \pm 1.68$ & $3.87-3.97$ \\
\hline & Settings & $6.90 \pm 2.22$ & $6.84-6.96$ \\
\hline & Weather & $2.37 \pm 1.40$ & $2.33-2.41$ \\
\hline
\end{tabular}

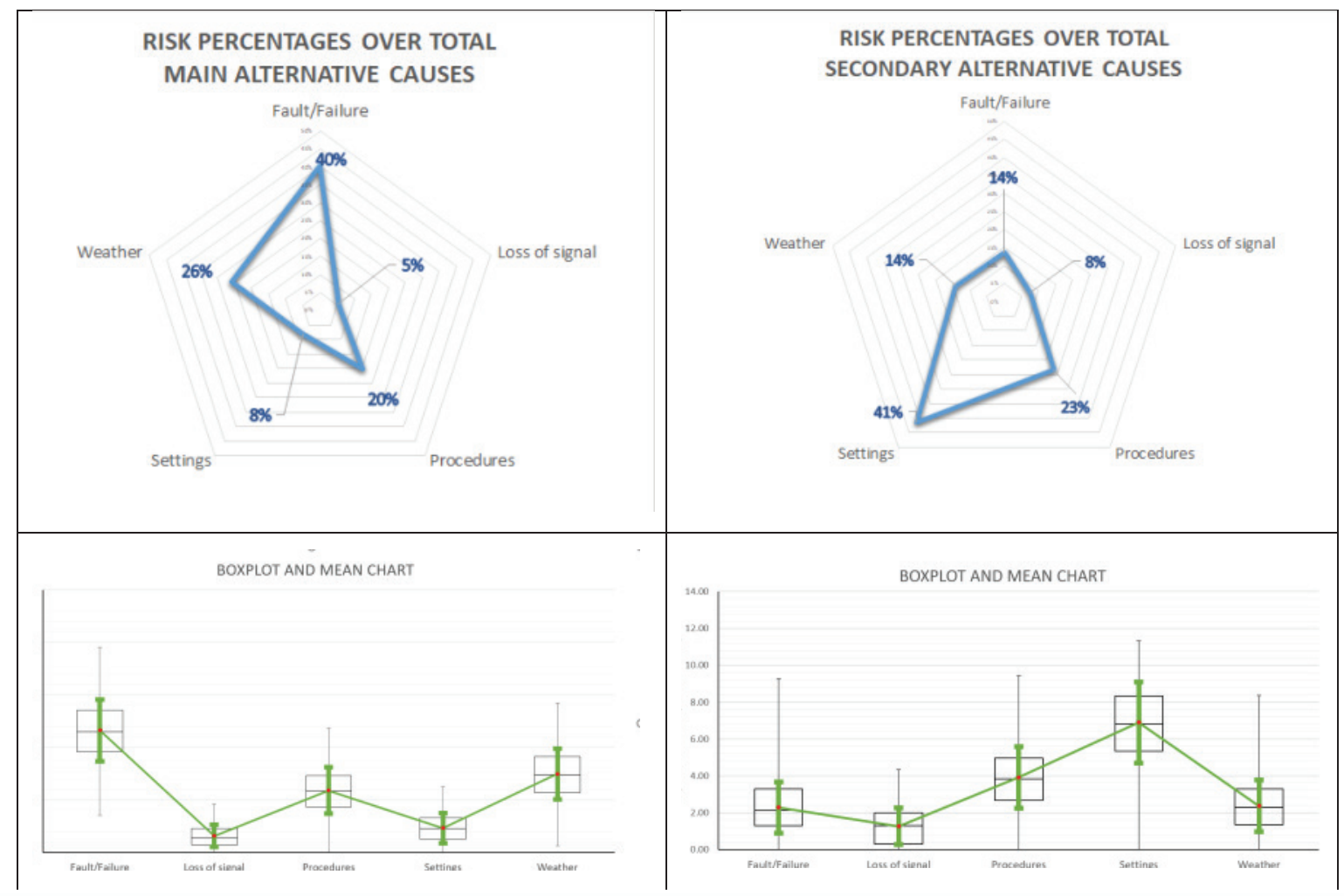

Figure 5 .

Top: Risk percentages for main alternative causes (left) and secondary alternative causes (right). Bottom: Boxplot and mean chart (showing standard deviations) for expected losses of main alternative causes (left) and secondary alternative causes (right). 


\section{DISCUSSION}

In the annual description of the database, it can be seen how the number of incidents is increasing. The severity of the consequences is higher at the beginning of the period, improving in the last years. However, this tendency could be influenced by the reporter's mentality to include those potentially dangerous incidents although there was not any great consequence. The company policies indicating the necessity of sending such reports could have influenced this trend.

The correlation between the traditional and alternative categorisation of causes shows the nature of the incidents for each segment of the dynamic positioning system. The results indicate that Environment causes are significantly due to weather conditions and Human errors have their origin mainly in procedures that are not followed correctly and the Referencescaused incidents because they lose their signals. Other segments are affected in more than one way.

At the same time, it is interesting to check the main causes of disruption for the different DP segments as the most significant percentage of faults and failures happen in the Power generation segment.

The secondary causes, when reported, add value to the incident and help define the origin of the main cause. Although the $\mathrm{p}$-values indicate that the correlations cannot be extrapolated within the $95 \%$ confidence interval, it becomes very noticeable how the Human secondary causes are adding to the incidents in a very high percentage.

The frequency of the different categories is a variable of high impact for this study. Within the traditional classification, Environment, Power and Thruster were the most frequent main causes. The secondary causes that occur most frequently are the ones caused by Human errors. Within the alternative classification, incidents caused by Faults or Failures are the main causes of the incidents, almost half of the total number of cases. They are followed by the incidents in which the Weather was the main cause. Regarding the secondary causes, wrong Settings and Procedures cover over half the cases.

When studying the consequences, the first step taken was to check whether a LoP had taken place or not. In this sense, the higher frequency for a LoP is found for Power causes. The higher frequency for a non-LoP incident was found to be for Thruster incidents. For the alternative classification, Fault/Failure and Loss of signal are not significantly predisposed to LoPs. In contrast, incidents caused by inadequate Procedures and Weather have a higher frequency of LoPs.

For the secondary causes, the external secondary cause had a more significant probability of having a LoP. The rest of the causes did not have the same significance, but power is within the secondary causes with a more significant probability of having a LoP. Taking into account the alternative classification, in this case there were no significant causes with a higher likelihood of LoP, the incidents caused by inadequate Procedures having the most significant percentage.

A fascinating discovery of the research was the significance of the results for the consequences based on the secondary causes when a LoP had taken place, as Power and External causes were the secondary causes with more significant possibilities of surpassing the red circle, followed by Human causes. For the alternative classification, Settings and Procedures are the secondary causes that mostly have a severe consequence and surpass the red circle.

The paper's main objective was to determine what kind of DP incidents are potentially more dangerous, having a higher risk. From the qualitative risk analysis results for the period, it can be determined that the Power-related incidents have the highest risk, with 8.37 million USD expected losses in 9 years, followed by Environmental and Human errors. The secondary causes with the higher expected losses are Human errors (3.60 million USD), followed by External and Power causes (3.27 million USD each).

In the alternative classification, the results are independent of each other. The incidents provoked by Faults or Failures are the riskiest ones, 11.64 million USD for the whole period (approximately 1.2 million USD expected losses per year). Weather and Procedures are the next-in-rank causes, having similar qualitative risks. Loss of signal is determined to be the least risky cause in this analysis. Regarding secondary causes, the highest risk appears to be settings, with a very significant mean value of 6.90 million USD for the period.

The distribution of the different causes, although it could not be determined to be normal or Poisson, has a positive skewness in common. The higher amount of incidents without LoP explains this fact. As this study is based on voluntary reports made by the members of the IMCA, it could be reasonable to think that a complete database in case the reporting of incidents were compulsory would add value to the results of the risk analysis.

\section{CONCLUSIONS}

Remarkably, most of the LoPs (53\%) had overpassed the red circle. This could indicate that the culture of reporting incidents still refers to reporting when something actually happens and not in the cases when all is resolved before any problematic or expensive situation develops.

By studying the LoPs and their different levels of danger, it was found out that Human error cases had the most significant probability of surpassing the red circle. Regarding the alternative classification, Faults and Failures were the causes with a more severe consequence of reaching the red circle.

Having all the above shreds of evidence taken into account, it can be determined that Power-caused incidents have a potentially more significant risk since the frequency is high and 
the consequences are severe. In the same way, incidents due to Environmental aspects are also considered to have a significant risk. However, Thruster events appear to have a lower risk even though their likelihood is high.

With the alternative classification, the incidents initiated by a fault or a failure have a higher risk. It could be concluded that the operators should beware of failures in the power system as this seems to be the combination with a higher risk.

Another aspect to consider is that Human factor, which does not appear to be the primary cause with a high frequency, often appears as a secondary cause. The consequences of the Human factor combined with inadequate Procedures, as shown by the alternative classification, have a potentially high risk that should be taken into account and corrected with more specific or specialised training.

In future research, the contributions of different factors such as the configuration of the system or meteorological conditions will be taken into account to build a model explaining the Human error contributions and help minimise them.

\section{REFERENCES}

Abrecht, B. R., 2016. Systems Theoretic Process Analysis applied to an Offshore Supply Vessel dynamic positioning system, M.I.T. Thesis, Massachusetts Institute of Technology, USA.

Adamson, A. and Abrahamsen, B., 2006. WSOG - History and Future. A summary of the Development \& Worldwide use of Well-Specific Operating Guidelines, MTS Dynamic Positioning Conference.

Ancione, G., Bragatto, P. \& Milazzo, M.F., 2020. A Bayesian network-based approach for the assessment and management of ageing in major hazard establishments. Journal of Loss Prevention in the Process Industries, 64, p.104080. Available at: http://dx.doi.org/10.1016/j.jp.2020.104080.

Bhalla, K. and Cao, Y., 2005. Watch Circle Assessment of Drilling Risers during a Drift-Off and Drive-Off Event of a Dynamically Positioned Vessel, MTS Dynamic Positioning Conference, pp. 15.

Bray, D., 2018a. DP Operations. Part 1 - Basic Principles and Systems, Seaways, August 2018, pp. 22-25.

Bray, D., 2018b. 'DP Operations. Part 2 - What is DP used for?, Seaways, September 2018, pp. 13-16.

Chae, C.-J., 2015. A Study on Human Error of DP Vessels LOP Incidents. Journal of the Korean Society of Marine Environment and Safety, 21(5), pp.515-523. Available at: http://dx.doi.org/10.7837/kosomes.2015.21.5.515.

Chae, C.-J., 2017. A Study on FSA Application for Human Errors of Dynamic Positioning Vessels Incidents, Journal of Korean Navigation and Port Research, 41 (5), pp. 259-268. Available at:

http://dx.doi.org/10.5394/KINPR.2017.41.5.259.

Chen, H., Moan, T. \& Verhoeven, H., 2008. Safety of dynamic positioning operations on mobile offshore drilling units. Reliability Engineering \& System Safety, 93(7), pp.1072-1090. Available at: http://dx.doi.org/10.1016/j.ress.2007.04.003.
Dong, Y., Vinnem, J.E. \& Utne, I.B., 2017. Improving safety of DP operations: learning from accidents and incidents during offshore loading operations. EURO Journal on Decision Processes, 5(1-4), pp.5-40. Available at:

http://dx.doi.org/10.1007/s40070-017-0072-1.

International Marine Contractors Association (IMCA), 2009. Dynamic Positioning Station Keeping Incidents - Incidents Reported for 2007. London: IMCA.

International Marine Contractors Association (IMCA), 2010. Dynamic Positioning Station Keeping Incidents - Incidents Reported for 2008. London: IMCA.

International Marine Contractors Association (IMCA), 2011. Dynamic Positioning Station Keeping Incidents - Incidents Reported for 2009. London: IMCA.

International Marine Contractors Association (IMCA), 2012. Dynamic Positioning Station Keeping Incidents - Incidents Reported for 2010. London: IMCA

International Marine Contractors Association (IMCA), 2015a. Dynamic Positioning Station Keeping Incidents - Incidents Reported for 2011. London: IMCA.

International Marine Contractors Association (IMCA), 2015b. Dynamic Positioning Station Keeping Incidents - Incidents Reported for 2012. London: IMCA.

International Marine Contractors Association (IMCA), 2015c. Dynamic Positioning Station Keeping Incidents - Incidents Reported for 2013. London: IMCA.

International Marine Contractors Association (IMCA), 2016a. Dynamic Positioning Station Keeping Incidents - Incidents Reported for 2014. London: IMCA.

International Marine Contractors Association (IMCA), 2016b. Dynamic Positioning Station Keeping Review - Incidents and Events Reported for 2015. London: IMCA.

International Marine Contractors Association (IMCA), 2016c. The Training and Experience of Key DP Personnel, Rev. 2. London: IMCA.

International Marine Contractors Association (IMCA), 2020. Guidelines for the design and operation of dynamically positioned vessels. London: IMCA.

International Marine Contractors Association (IMCA), 2021. M 220 Rev. 2 Guidance on Operational Activity Planning. London: IMCA.

International Maritime Organisation, 1994. Guidelines for Vessels with Dynamic Positioning Systems.

International Maritime Organisation, 2009. Code for the construction and equipment of Mobile Offshore Drilling Units.

International Maritime Organisation, 2011. International Convention on Standards of Training, Certification and Watchkeeping for Seafarers (STCW).

International Maritime Organisation, 2017a. Guidelines for Dynamic Positioning (DP) Operator Training.

International Maritime Organisation, 2017b. Guidelines for Vessels and Units with Dynamic Positioning (DP) Systems.

Khan, F.I. \& Abbasi, S., 1998. Techniques and methodologies for risk analysis in chemical process industries. Journal of Loss Prevention in the Process Industries, 11(4), pp.261-277. Available at:

http://dx.doi.org/10.1016/s0950-4230(97)00051-x.

Kristiansen, S., 2005. Part III. Risk Analysis' in Butterworth-Heinemann (eds.) Maritime Transportation. Safety Management and Risk Analysis, New York: Elsevier.

Leveson, N.G. et al., 2012. Safety Assessment of Complex, Software-Intensive Systems. SAE International Journal of Aerospace, 5(1), pp.233-244. Available at: http://dx.doi.org/10.4271/2012-01-2134. 
Marine Technology Society, 2012. DP Operations Guidance - Part 2 - Appendix 1 MODUs.

Marine Technology Society, 2021. Marine Technology Society [Online]. Available at: https://www.mtsociety.org, accessed on: 09 January 2021.

Norwegian Shipowners' Association, Norwegian Oil and Gas Association, Netherlands Oil \& Gas Production Association, Danish Shipowners Association, Oil \& Gas UK \& United Kingdom Chamber of Shipping (2009) Guidelines for the Safe Management of Offshore Supply and Rig Move Operations (NW European Area) (NWEA Guidelines).

Norwegian Shipowners' Association, Norwegian Oil and Gas Association, Netherlands Oil \& Gas Production Association, Danish Shipowners Association, Oil \& Gas UK \& United Kingdom Chamber of Shipping (2013) Guidelines for Offshore Marine Operations (G-OMO).

Øvergård, K.I. et al., 2015. Critical incidents during dynamic positioning: operators situation awareness and decision-making in maritime operations. Theoretical Issues in Ergonomics Science, 16(4), pp.366-387. Available at: http://dx.doi.org/10.1080/1463922x.2014.1001007.

Parhizkar, T. et al., 2020. Data driven approach to risk management and decision support for dynamic positioning systems. Reliability Engineering \& System Safety, 201, p.106964. Available at:

http://dx.doi.org/10.1016/j.ress.2020.106964.
Quigley, C. \& Williams, D., 2015. A Revised Methodology for the Calculation of MODU Watch Circles. Volume 5A: Pipeline and Riser Technology. Available at: http://dx.doi.org/10.1115/omae2015-41230.

Sanchez-Varela, Z. et al., 2021. Prediction of Loss of Position during Dynamic Positioning Drilling Operations Using Binary Logistic Regression Modeling. Journal of Marine Science and Engineering, 9(2), p.139. Available at: http://dx.doi.org/10.3390/jmse9020139.

Seabrokers Ltd, A., 2012-2015. Seabreeze, Aberdeen, UK: Seabrokers Ltd:

Sulaman, S.M. et al., 2017. Comparison of the FMEA and STPA safety analysis methods-a case study. Software Quality Journal, 27(1), pp.349-387. Available at: http://dx.doi.org/10.1007/s11219-017-9396-0.

Teixeira, F.J.R., Oshiro, A.T. and Tannuri, E.A., 2014. Drifting Time of a Standard Drillship, New York, USA: Amer Soc Mechanical Engineers.

Verhoeven, H., Chen, H. and Moan, T., 2006. Safety of Dynamic Positioning Operation on Mobile Offshore Drilling Units, MTS Dynamic Positioning Conference.

Weingarth, L., 2006. Refining the DP Watch Circle, MTS Dynamic Positioning Conference. 